\title{
Association between HPV and Head and Neck Cancer: Differences in Understanding among Three Distinct Populations
}

\author{
Laura J. White ${ }^{1}$, Francis X. Creighton $\mathrm{Jr}^{2}$, Justin C. Wise ${ }^{3}$, Edie R. Hapner ${ }^{1, *}$ \\ ${ }^{1}$ Department of Otolaryngology Head and Neck Surgery, Emory University School of Medicine, Atlanta, GA 30308 \\ ${ }^{2}$ Massachusetts Eye and Ear Infirmary, Boston, MA 02114 \\ ${ }^{3}$ Oglethorpe University, Atlanta, GA 30319 \\ *Corresponding author: ehapner@emory.edu
}

Received March 26, 2013; Revised May 28, 2013; Accepted February 09, 2014

\begin{abstract}
The aim of this study is to compare the knowledge of the relationship between HPV and head and neck cancer (HNCA) in three unique populations: general public at a sporting event, undergraduates, and medical students, and to compare the frequency that a healthcare provider has discussed the topics of cervical cancer and HNCA amongst these populations. A one-way between subjects design was used to evaluate participants at a community based head and neck cancer screening, and students in individual classrooms. A28-item questionnaire was administered to 491 NASCAR race attendees, 186 undergraduate students, and 158 medical students. The survey assessed their knowledge of the relationship between HPV and HNCA using a Likert scale. The survey also assessed the level of information disseminated by healthcare providers regarding HPV and HNCA. NASCAR attendees evidenced significantly $\left(\mathrm{p}<.001, \eta^{2}=.10\right)$ lower levels of knowledge of the relationship between HPV and cervical cancer than college or medical students. NASCAR attendees evidenced significantly $\left(p<.001, \eta^{2}=.02\right)$ lower levels of knowledge of the relationship between HPV and HNCA. NASCAR attendees reported significantly $\left(p<.001, \eta^{2}\right.$ $=.10$ ) more frequent discussions with health care providers regarding HNCA compared to college or medical students. Undergraduates and medical students reported significantly $\left(\mathrm{p}<.001, \eta^{2}=.02\right)$ more frequent discussions with health care providers regarding HPV than NASCAR attendees. Results demonstrated group differences in knowledge between the relationship of HPV and cervical cancer and HPV and HNCA. All groups were more aware of HPV's association with cervical cancer than with HNCA.
\end{abstract}

Keywords: adult, head and neck carcinoma, patient understanding, HPV

Cite This Article: Laura J. White, Francis X. Creighton Jr, Justin C. Wise, and Edie R. Hapner, "Association between HPV and Head and Neck Cancer: Differences in Understanding among Three Distinct Populations." American Journal of Cancer Prevention, vol. 2, no. 1 (2014): 14-19. doi: 10.12691/ajcp-2-1-5.

\section{Introduction}

There has been a dramatic rise in the number of Human Papilloma Virus (HPV)-positive oropharyngeal cancers in the United States. [1] Although HPV can be present in other sites, there is a predilection for the oropharynx and approximately $50 \%$ of all cases of oropharyngeal cancer are positive for HPV. [2] As the symptoms of oropharyngeal cancer are largely non-specific, many patients present with advanced disease that is associated with a poorer prognosis. [3] In a large study of tonsillar cancer, $65 \%$ of patients presented with Stage IV disease. [4] It has been shown that NASCAR attendees, a population with higher than average smoking rates and concerning findings on head and neck cancer screening exams, have low levels of knowledge and worry about HNCA [5].

The prevalence of oral HPV infection is $6.9 \%$ and that of HPV-16 is 1\%. [2] Oral HPV infection is more common among men, with a prevalence of $10.1 \%$ and
HPV-16 prevalence of $1.6 \%$. Those who are positive for oral HPV infection are more likely to be current smokers, started oral sexual activity at a younger age, had greater number of sex partners, and a history of oral-genital sex. $[6,7,8]$ Additionally, open-mouthed kissing is associated with the development of oral HPV infection. Those with oral HPV are more likely to have had more than 20 lifetime vaginal sex partners and greater than 6 oral sex partners.

HPV-16 and HPV-18 are responsible for $70 \%$ of all new cases of cervical cancer. [9] Prevention of cervical cancer includes primary prevention through the HPV vaccination and secondary prevention through cervical cancer screening with the Papanicalaou (Pap) test. A quadravalent vaccine protecting against HPV types 16, 18, 6 , and 11 became widely available and commercialized beginning in 2006. The US Centers for Disease Control and Prevention recommend the vaccine be administered to females and males aged 9 to 26 to prevent genital warts and anogenital cancers. [10] In a recent study examining general knowledge of HPV in the post-vaccine era, 97\% 
of college females had heard of HPV and 84\% knew that there was a link between HPV and cervical cancer. [11] Very few studies included men's perceptions and understanding of HPV as it relates to cervical cancer. The effectiveness of the HPV vaccine against oral HPV infection is unknown and vaccination is not currently recommended for the primary prevention of oropharyngeal cancer. [12] There is no standardized screening test like Pap test available for oral HPV.

There is an abundance of information regarding women's understanding of HPV and it's association with cervical cancer, yet there is little data examining the layperson's understanding of the association between HPV and oropharyngeal cancer. As the risk factors for oropharyngeal HPV include commonplace activities such as kissing, it is important for the general population to be aware of the risk factors. The highest prevalence of HPV infection is among those aged 14-19; therefore, undergraduate students are at high risk of HPV infection and HPV-related complications. It has been shown that accurate knowledge of HPV infection is necessary to make appropriate, evidence-based choices among prevention strategies, including vaccination and routine screening. [13] There is no data concerning college students' understanding of the association between HPV and oropharyngeal cancer. It is a physician's responsibility to accurately counsel patients regarding cancer risk and screening guidelines to allow them to make evidencebased choices. [14] However, it has been shown that medical students lack information regarding HNCA risk factors, oral screening guidelines, and head and neck examination techniques. $[15,16,17]$ These studies did not examine medical student knowledge about HPV as a risk factor for head and neck cancer.

Therefore, this study aimed to (1) compare the knowledge of the relationship between HPV and head and neck cancer (HNCA) and HPV and cervical cancer in three unique populations: participants at a large sporting event, undergraduate students, and medical students and to (2) compare the knowledge of the mode of transmission of HPV amongst three unique populations and to (3) compare the self-reported frequency that a healthcare provider has discussed the topics of cervical cancer versus HNCA and their relation to HPV amongst three unique populations.

\section{Methods}

Emory University Institutional Review Board approval was obtained. This study was survey-based using convenience sampling to recruit the study sample from a community identified as at-risk of developing head and neck cancer as well as from two student populations. Prior to beginning the study, a power analysis was performed. With a power of .80 , an alpha of .05 , and a small effect size, power analysis indicated that 145 participants per group were needed to test for significant differences among groups.

\subsection{Participants}

Community participants were recruited from NASCAR fans touring the midway before entry into the race venue at Atlanta Motor Speedway in Hampton, GA during a race weekend event. Undergraduate students from Oglethorpe
University and University of Georgia as well as medical students from Emory University were recruited from their classrooms.

At the NASCAR race, male and female participants were randomly selected from an estimated two hundred thousand fans touring the midway during the weekend. All race attendees and students age 18 and older were eligible to participate.

\subsection{Materials and Procedures}

The questionnaire developed for this study was adapted from items on validated questionnaires that evaluated smokers' risk perceptions as well as previous knowledgebased questionnaires used by the authors' to investigate the NASCAR cohort. [5,18,19,20] The wording of some items was changed so that the language was accessible to the wide range of literacy levels anticipated in the study populations.

The final questionnaire was a 28-item survey that collected demographic information, highest education level achieved, smoking status, and awareness of the relationship between HPV and cervical cancer and HNCA, and awareness of how HPV is transmitted (Appendix A). Participants orally consented to complete the survey after receiving an information sheet regarding the study aims.

Pearson chi-square tests were used to test for demographic group differences with dichotomous data, Independent sample t-tests were used to test for group differences with independent quantitative outcome variables, and multivariate analysis of covariance (MANCOVA) analyses were used to test for group differences in the in the presence of multiple, related dependent variables. All tests were 2-tailed and p-values less than 0.05 were considered significant.

\section{Results}

Eight hundred thirty-seven total participants completed the questionnaire. This included 491 NASCAR attendees, 158 medical students, and 186 undergraduate students. Additional demographic information is outlined in Table 1. Because of the nature of the data, significant differences between groups for the question, 'who is affected by HPV?', with the options men, women, both men and women, or I don't know are not reported. However, descriptively, $60.2 \%$ of NASCAR attendees, $71.0 \%$ of undergraduates, and $83.5 \%$ of medical students answered that HPV affects both men and women.

Table 1. Demographic details of the three individual populations

\begin{tabular}{lccc}
\hline & $\begin{array}{c}\text { NASCAR } \\
(\mathrm{n}=491)\end{array}$ & $\begin{array}{c}\text { Medical Students } \\
(\mathrm{n}=158)\end{array}$ & $\begin{array}{c}\text { Undergraduates } \\
(\mathrm{n}=186)\end{array}$ \\
$\begin{array}{l}\text { GENDER (\%) } \\
\text { Male }\end{array}$ & 45.9 & 45.5 & \\
$\quad$ Female & 54.1 & 54.5 & 76.8 \\
ETHNICITY & & & \\
(\%) & & & \\
White & 86.5 & 63.3 & 61.3 \\
Black & 7.8 & 8.9 & 19.4 \\
Asian & 1.6 & 19.6 & 3.2 \\
Biracial & 1.0 & 1.3 & 7.0 \\
Hispanic & 2.0 & 2.5 & 7.5 \\
Other & 1.0 & 4.4 & 1.6 \\
AGE (M +/- & & & \\
SD) & $23.83+/-$ & $37.67+/-13.92$ & $20.33+/-3.95$ \\
& 2.14 & &
\end{tabular}


Of the NASCAR fans, $14.5 \%$ had attended college and $34.3 \%$ had a bachelor's degree or higher. A Pearson correlation analysis was conducted to determine if there was a relationship between participants' level of education, gender, or age and knowledge of HPV and HNCA, HPV and cervical cancer, or and how HPV is transmitted within the group of NASCAR attendees. No significant correlations were found between level of education, gender, or age and these variables $(p>.05)$ and therefore they were not used as a covariate in further analyses. Because the undergraduates and medical students had at least obtained a college education and a graduate education, respectively, and age range was so similar, differences in education and age were not reported. Due to the limited racial diversity among the populations, subtype analysis based on race was not performed.

Two independent MANOVA analyses were conducted to test for group differences among undergraduates, NASCAR fans, and medical students. The first MANOVA tested group differences for the risk perception variables of how much do you agree that HPV increases the risk of
HNCA; how much do you agree that HPV increases the risk of cervical cancer; and how much do you agree that increased numbers of sexual partners increases the risk of $\operatorname{HPV}$, with $\mathrm{F}(6,1638)=21.72, \mathrm{p}<.001, \eta 2=.07$. The MANOVA was significant, therefore follow-up univariate analysis of variance for each independent variable was warranted.

Analyses indicated a significant main effect among groups for all three dependent variables. Please refer to Table 2 for the specific values. Tukey post-hoc analysis demonstrated that medical students were significantly more aware of the association between HPV and HNCA than both NASCAR attendees and undergraduates. Tukey post-hoc analysis showed that medical students were significantly more aware of the association between HPV and cervical cancer than both undergraduates and NASCAR attendees. Finally, Tukey post-hoc analysis demonstrated that medical students were more aware that increased sexual partners increases the risk of HPV than both undergraduates and NASCAR attendees.

Table 2. Individual Tukey Post-hoc analysis of group differences among Medical Students, Undergraduate Students, and NASCAR attendees

\begin{tabular}{|c|c|c|c|c|c|c|c|c|c|}
\hline & \multicolumn{3}{|c|}{ Medical Students } & \multicolumn{2}{|c|}{ Undergraduates } & \multicolumn{2}{|c|}{ NASCAR } & \multirow[b]{2}{*}{$\eta 2$} & \multirow[b]{2}{*}{$\mathbf{F}$} \\
\hline & $\mathrm{p}$ & Mean & SD & Mean & SD & Mean & SD & & \\
\hline Does HPV increase the risk of HNCA? & $<.001$ & 2.84 & 1.07 & 2.31 & 0.93 & 2.63 & 1.12 & 0.03 & 12.24 \\
\hline Does HPV increase the risk of cervical cancer? & $<.001$ & 3.85 & 0.36 & 3.46 & 0.73 & 3.13 & 0.99 & 0.10 & 31.45 \\
\hline $\begin{array}{l}\text { Do increased numbers of sexual partners increase } \\
\text { the risk of HPV? }\end{array}$ & $<.001$ & 3.73 & 0.54 & 3.38 & 0.82 & 3.08 & 0.99 & 0.07 & 25.67 \\
\hline
\end{tabular}

An additional 3 (group) X 2 (gender) MANOVA was conducted for the dependent variables of how much do you agree that HPV is spread through intercourse, how much do you agree that HPV is spread through kissing; how much do you agree that HPV is spread through oral sex, how often has a provider talked to you about HNCA; and how often has a provider talked to you about HPV.
Analyses revealed that there was a significant effect for group for each question with $F(10,1598)=p<.001, \eta 2$ $=.12$. Therefore, individual follow up ANOVAS were performed. Analyses indicated a significant main effect among groups for all three dependent variables. Please refer to Table 3 for the specific values.

Table 3. Individual Tukey post-hoc analysis as follow-up of 3 (group) X 2 (gender) MANOVA for group differences among Medical Students, Undergraduate Students, and NASCAR attendees

\begin{tabular}{|c|c|c|c|c|c|c|c|c|c|}
\hline & \multicolumn{3}{|c|}{ Medical Students } & \multicolumn{2}{|c|}{ Undergraduates } & \multicolumn{2}{|c|}{ NASCAR } & \multirow[b]{2}{*}{$\eta 2$} & \multirow[b]{2}{*}{$\mathbf{F}$} \\
\hline & $\mathrm{p}$ & Mean & SD & Mean & SD & Mean & SD & & \\
\hline $\begin{array}{l}\text { Can HPV be spread through sexual } \\
\text { intercourse? }\end{array}$ & $<.001$ & 3.81 & 0.44 & 3.35 & 0.92 & 2.95 & 1.09 & 0.12 & 22.84 \\
\hline Can HPV be spread through kissing? & $<.001$ & 1.41 & 1.10 & 1.41 & 1.06 & 1.83 & 1.23 & 0.24 & 11.14 \\
\hline $\begin{array}{l}\text { Can HPV be spread through oral } \\
\text { sex? }\end{array}$ & $<.001$ & 3.21 & 0.92 & 2.76 & 1.02 & 2.58 & 1.10 & 0.05 & 9.15 \\
\hline $\begin{array}{l}\text { How often has a healthcare provider } \\
\text { talked to you about HNCA? }\end{array}$ & $<.001$ & 0.43 & 0.84 & 0.42 & 0.78 & 1.10 & 1.14 & 0.09 & 17.13 \\
\hline $\begin{array}{l}\text { How often has a healthcare provider } \\
\text { talked to you about HPV? }\end{array}$ & .001 & 1.39 & 1.10 & 1.34 & 1.08 & 1.01 & 1.08 & 0.02 & 44.09 \\
\hline
\end{tabular}

Tukey post-hoc analysis showed that medical students were significantly more aware that HPV was transmitted via sex than undergraduates and NASCAR attendees. Tukey post-hoc analysis demonstrated that NASCAR attendees were significantly more aware that HPV is transmitted via kissing than both undergraduates and medical students. Tukey post-hoc analysis demonstrated that medical students were significantly more aware that HPV is transmitted via oral sex than both undergraduates and NASCAR attendees. Tukey post-hoc analysis demonstrated that healthcare providers had spoken to NASCAR attendees about HNCA significantly more than both medical students and undergraduates. Tukey post-hoc analysis demonstrated that healthcare providers had spoken to undergraduates and medical students significantly more than NASCAR attendees about HPV.

MANOVA analysis also demonstrated a significant main effect for gender with $F(5,798)=p<.001, \eta 2=.25$. Therefore, individual follow up ANOVAs were performed. For the question, “is HPV spread through sex?", there was a significant main effect of gender, $F(1,802)=p=.004$, $\eta 2=.01$. Females $(\mathrm{M}=3.32, \mathrm{SD}=0.99)$ were significantly more aware that HPV is transmitted via sex than males $(\mathrm{M}=3.02, \mathrm{SD}=1.04)$. For the question, "is HPV spread through kissing?”, there was significant main effect of gender, $F(1,802)=p<.001, \eta 2=.03$. Males $(M$ 
$=1.92, \mathrm{SD}=1.13)$ were significantly more aware that $\mathrm{HPV}$ is transmitted via kissing than females $(\mathrm{M}=1.47$, $\mathrm{SD}=1.09$ ). For the question, "is HPV spread by oral sex?", there was a significant main effect of gender, $F$ $(1,802)=\mathrm{p}=.038, \eta 2=.005$. Females $(\mathrm{M}=2.80, \mathrm{SD}=$ 1.11) were significantly more aware that HPV is transmitted via oral sex than males $(\mathrm{M}=2.65, \mathrm{SD}=1.01)$. For the question, "how often has a healthcare provider spoken to you about HNCA?”, there was no significant main effect of gender $(p>.05)$. For the question, "how often has a healthcare provider spoken to you about HPV?", there was a significant main effect of gender $\mathrm{F}(1,802)=\mathrm{p}<.001, \eta 2=.19$. Healthcare providers spoke to females about HPV $(\mathrm{M}=1.54, \mathrm{SD}=1.07)$ significantly more than males $(\mathrm{M}=0.60, \mathrm{SD}=0.87)$.

MANOVA analysis also showed a significant gender $\mathrm{X}$ group interaction, $F(10,1598)=p .001, \eta 2=.023$. Follow-up univariate ANOVAs demonstrated only one significant interaction among the multiple dependent variables, $F(2,802)=p<0.001, \eta 2=.026$. Healthcare providers had spoken to males within the NASCAR and medical groups slightly more than the undergraduate group. However, for females, healthcare providers had spoken to the medical school group much more frequently than undergraduates and even more so compared to NASCAR attendees, (see Figure 1).

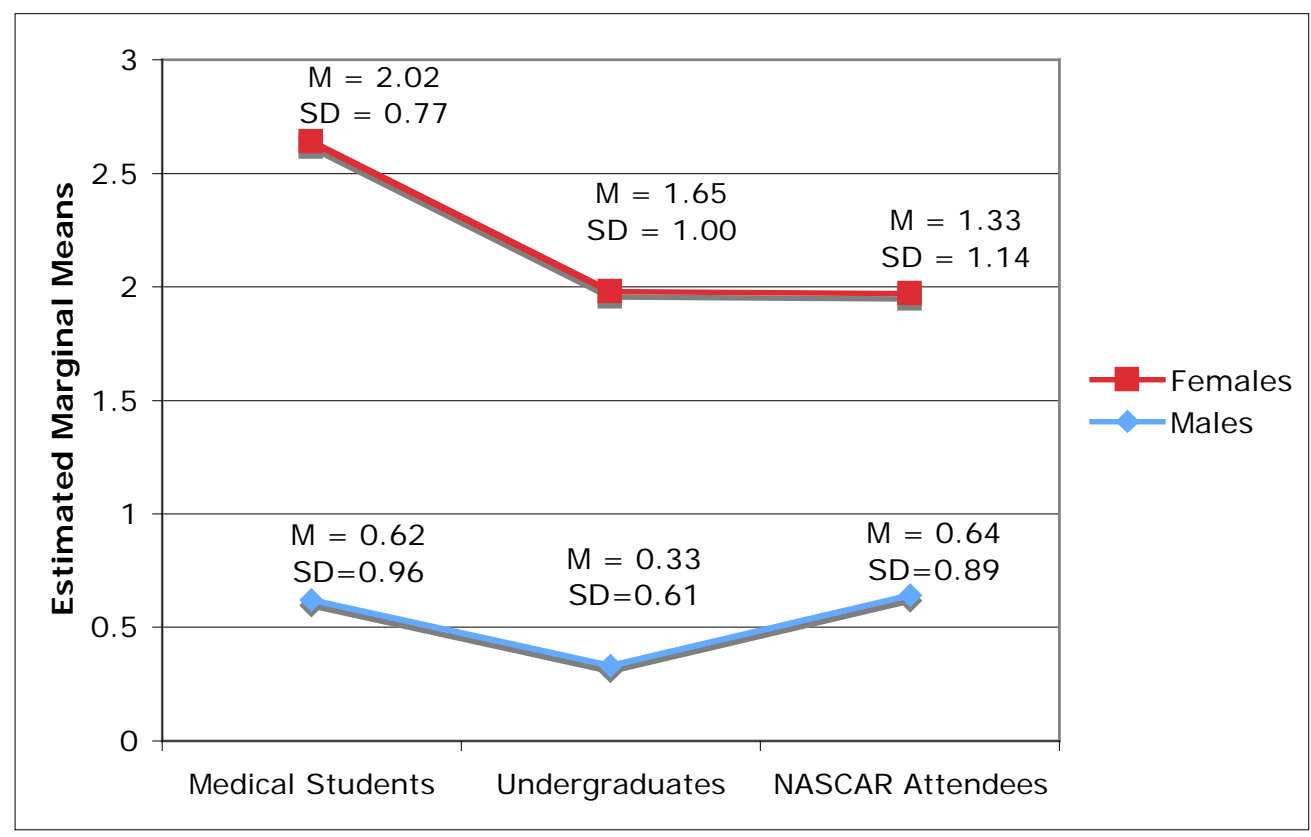

Figure 1. Univariate gender $\mathrm{X}$ group ANOVA testing group differences among Medical Students, Undergraduates, and NASCAR attendees for the question, How often has a healthcare provider spoken to you about HPV?

\section{Discussion}

This study was designed to examine differences in the understanding of the relationship between $\mathrm{HPV}$ and HNCA, HPV and cervical cancer, and knowledge of the mode of transmission of HPV amongst the three distinct populations: participants at a sporting event, undergraduate students, and medical students, as well as to compare the self-reported frequency that a healthcare provider had discussed the topic of cervical cancer and HNCA in these populations. Although the results were significant amongst groups, the effect size was very small, indicating general agreement between groups. Therefore, the trends are more important than individual differences when interpreting the results.

All groups were more aware of the association between HPV and cervical cancer than HPV and HNCA, including medical students. The high level of awareness of HPV and cervical cancer is consistent with previous studies. ${ }^{11}$ This is likely because the association between HPV and cervical cancer has been widely publicized. After the quadrivalent HPV vaccine was licensed for use in the United States in 2006, Merck's '1 less' campaign aired in 2008 on both TV and in print and clearly stated that the HPV vaccine decreased a woman's risk of developing cervical cancer. There has not been a similar national media campaign outlining the association between HPV and oropharyngeal cancer.

All groups were aware that increased numbers of sexual partners leads to increased risk of HPV infection. This is consistent with all groups being aware that HPV is transmitted through sexual intercourse. However, there was low awareness of the association between HPV and oral sex and even more so with HPV and deep kissing. This is especially true amongst medical students and undergraduate students, who disagreed that there was an association. Once again, this is likely due to the widely publicized nature of HPV as a sexually transmitted disease leading to cervical cancer and the lack of publicized information regarding oropharyngeal HPV.

All groups reported that healthcare providers had spoken to them infrequently about both HPV and HNCA. Additionally, across all groups, providers had spoken to participants more about HPV than HNCA. This may be due to lack of knowledge amongst healthcare providers about HNCA. Medical students had a lack of knowledge regarding the association between HPV and HNCA. This is consistent with previous studies demonstrating lack of knowledge of head and neck cancer amongst medical students. This has been related to a lack of information regarding HNCA presented in the medical school 
curricula. $[15,16,17]$ If our future healthcare providers are unaware of the association between HPV and HNCA, they cannot adequately counsel and educate their patients regarding its risks, signs, and symptoms.

In this study, nearly $60 \%$ of respondents were female. Many of the previous studies assessing patient knowledge of HPV surveyed only women and examined HPV knowledge relating to cervical cancer and HPV vaccination. [11] In this study, healthcare providers had spoken to women about HPV significantly more than men. However, healthcare providers had not spoken to either group very often, with women being at the midpoint of the scale (1.5) and men being far below the midpoint (0.6). Additionally, women were more aware than men that HPV is spread via oral sex and vaginal sex. Although women had more knowledge of HPV transmission than men, differences were small with a small effect size. Therefore, the difference is not likely explained by the increased information provided by healthcare providers.

Despite the findings of group differences, all groups were more aware of the association between HPV and cervical cancer than HPV and HNCA. Additionally, healthcare providers are not speaking to any of these groups about HPV or HNCA. Especially concerning is that medical students were unaware that HPV can be transmitted via kissing. If the future healthcare providers lack knowledge regarding HPV and HNCA, they will not be able to educate their patients.

\subsection{Limitations}

The undergraduate students were attendees of a small, private university in the southeast and may not be representative of undergraduate students at larger, state universities. Similarly, the medical students were recruited from a private university in the southeast and may not be representative of all medical students.

\section{Conclusion}

All of the groups surveyed were less aware of HPV and HNCA than HPV and cervical cancer and indicated that healthcare providers were not providing information regarding HNCA and HPV to them. Although these groups were not demographically representative of the general population, given that none of these participants, including medical students were aware of the association between HPV and HNCA, it is unlikely that the average person is. Otolaryngologists must make an effort to educate their patients about the risks of HNCA, including $\mathrm{HPV}$, in the clinic setting. However, the reality is that few people see the otolaryngologist without referral from other healthcare professionals, often presenting a significant lapse in time and opportunity for early diagnosis of head and neck cancer. Therefore, otolaryngologists are not the front line providers of care that must be targeted for education initiatives. It seems incumbent on primary care physicians, dentists, nurse practitioners and other front line medical personnel to provide education regarding the signs, symptoms and risks of HNCA. Additionally, the community needs to be educated. The use of communitybased events presents another opportunity for widespread education and screening. Educating medical, dental, nursing students, and the public itself appears pivotal to making a difference in reducing the 30 year trend of later diagnosis of this disease with increased morbidity and mortality.

\section{Competing Interests}

Senior author, Edie Hapner, is a national board member of A Voice for Hope: United in the Battle Against Mouth and Throat Cancer, 501(c)3.

\section{References}

[1] Chaturvedi AK, Engels EA, Pfeiffer RM, et al. Human papillomavirus and rising oropharyngeal incidence in the United States. J Clin Oncol. 2001; 29(32);4294-4301.

[2] Ernster JA, Sciotto CG, O’Brien MM, et al. Rising incidence of oropharyngeal cancer and the role of oncogenic human papilloma virus. Laryngoscope. 2007; 117:2115-2128.

[3] Rogers SN, Shreya VV, Lowe D. Reasons for delayed presentation in oral and oropharyngeal cancer: the patients perspective. Brit J Oral Max Surg. 2011;49(5):349-353.

[4] Attner P, Nasman A, Du J, et al. Survival in patients with human papillomavirus positive tonsillar cancer in relation to treatment. Int J Cancer. 2012; 131:1124-1130.

[5] White LJ, Chin-Quee AL, Berg CJ, et al. Differences in head and neck cancer risk perception between smoking and non-smoking NASCAR attendees. Otolaryngol Head Neck Surg. 2012;147:63-8.

[6] Smith, EM, Ritchie JM, Summersgill KF, et al. Age, sexual behavior, and human papillomavirus infection in oral cavity and oropharyngeal cancers. Int J Cancer 2004;108:766-772.

[7] Schwartz SM, Daling JR, Doody DR, et al. Oral cancer risk in relation to sexual history and evidence of human papillomavirus infection. J Natl Cancer Inst. 1998;90:1626-1636.

[8] D’Souza G, Agrawal Y, Hapern J, et al. Oral sexual behaviors associated with prevalent oral human papillomavirus infection. J Infect Disease. 2009; 199:1263-1269.

[9] Hariri S, Unger ER, Sternberg M, et al. Prevalence of genital human papillomavirus among females in the United States, the National Health and Nutrition Examination Survey, 2003-2006. J Infect Dis. 2011; 204(4):566-573.

[10] Centers for Disease Control and Prevention. 2010 Sexually Transmitted Diseases Surveillance.

http://www.cdc.gov/std/stats10/other.htm, accessed August 10, 2012.

[11] Gerend MA, Shepherd JE. Correlates of HPV knowledge in the Era of HPV vaccination: A study of unvaccinated young adult women. Women Health. 2011; 51(1):25-40.

[12] Centers for Disease Control and Prevention. Advisory Committee on Immunization Practices. Grading of recommendations, assessment, development, and evaluation for HPV vaccine in males. MMWR. 2011; 60:1705-8.

[13] Tiro JA, Meissner, HI, Kobrin S, Chollette V. What do women in the U.S. know about Human Papillomavirus and cervical cancer? Cancer Epidemio Biomarkers Prev. 2007; 16:288-294.

[14] Wolf AMD, Becker DM. Cancer screening and informed patient discussions. Arch Intern Med. 1996: 156(10):1069-1072.

[15] Mohyuddin N, Langerman A, LeHew C, et al. Knowledge of head and neck cancer among medical students at 2 Chicago Universities. Arch Otolaryngol Head Neck Surg. 2008:134(12):1294-8.

[16] Wu EC, Passy V, Armstrong WB. Preliminary evaluation of junior medical students' comfort with performing the basic head and neck examination. Laryngoscope. 2011;121:1431-1435.

[17] Ferullo A, Silk H, Savageau JA. Teaching oral health in US medical Schools: Results of a national survey. Acad Med. 2011; 86:226-230.

[18] Park ER, Ostroff JS, Rakowski W, et al. Risk perceptions among participants undergoing lung cancer screening: baseline results from the National Lung Screening Trial. Ann Behav Med. 2009; 37(3):268-79.

[19] Viscusi, WK. Age variations in risk perceptions and smoking decisions. Rev Econ Statist. 1991;73:577-588.

[20] Viscusi, WK. Do smokers underestimate risk? J Polit Economy. 1990;98:1253-1269. 


\section{APPENDIX A. Questionnaire}

1. What is your gender? (Choose one.)

2. How old are you? years old

3. In the past 30 days, on how many days did you smoke a cigarette?

4. What is your ethnicity? (choose one)

[ ] Asian or Pacific Islander

[ ] Biracial or Multiracial

5. What is your education level? (choose one)

[ ] Attended high school

[ ] Associates/Technical Degree

6. Smoking cigarettes increases the

Strongly Disagree Disagree

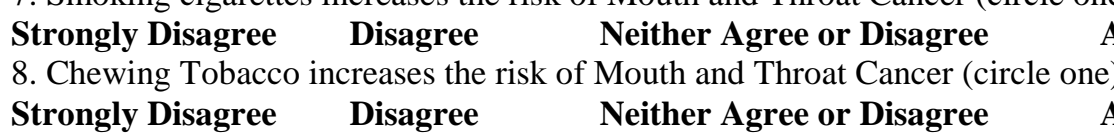
Agree Strongly Agree

9. HPV (Human Papilloma Virus infection increases the risk of Mouth and Throat Cancer (circle one)
Strongly Disagree
Disagree
Neither Agree or Disagree
Agree Strongly Agree

10. HPV (Human Papilloma Virus infection increases the risk of Cervical Cancer (circle one)
Strongly Disagree
Disagree
Neither Agree or Disagree
Agree Strongly Agree

11. Smoking cigarettes AND drinking alcohol increases the risk of Head and Neck Cancer more than smoking cigarettes alone (circle one)

Strongly Disagree Disagree $\quad$ Neither Agree or Disagree Agree Strongly Agree

12. You can get infected with HPV (Human Papilloma Virus) through sexual intercourse (circle one)

Strongly Disagree Disagree Neither Agree or Disagree Agree Strongly Agree

13. You can get infected with HPV (Human Papilloma Virus) through kissing (circle one)
Strongly Disagre
Disagree
Neither Agree or Disagree
Agree Strongly Agree

14. You can get infected with HPV (Human Papilloma Virus) through oral sex (circle one)
Strongly Disagree
Disagree
Neither Agree or Disagree
Agree Strongly Agree

15. Increasing numbers of sexual partners increases the risk of HPV (Human Papilloma Virus) (circle one)
Strongly Disagree
Disagree
Neither Agree or Disagree
Agree Strongly Agree

16. HPV is an infection that affects (circle one)

Men Women Both men and women

I Don't know

17. How often has a healthcare provider talked to you about cancer of the mouth and throat (circle one)?
Never
Rarely
Sometimes
Ofte

18. How often has a healthcare provider talked to you about Human Papilloma Virus (HPV) (circle one)?
Never
Rarely
Sometimes
Often

19. The fastest growing cause of cancer of the mouth and throat is (circle one)?

Smoking Chewing Tobacco HPV Infection None of the above

20. What level of knowledge do you have about cancer of the mouth and throat (circle one)?

None Very Little A Small Amount Good Very Good

21. How much do you worry about getting mouth, tongue, or throat cancer? (Circle one)

Not at all A little Somewhat Quite a lot Extremely

22. How dangerous is mouth, tongue, or throat cancer? (Circle one.)

$\begin{array}{llll}\text { Not at all } & \text { A little } & \text { Somewhat } & \text { Quite a lot }\end{array}$ 